\title{
JMJD6 exerts function in neuropathic pain by regulating NF- $\kappa$ B following peripheral nerve injury in rats
}

\author{
CEN WEN, MENGYUAN XU, CHENG MO, ZHIGANG CHENG, QULIAN GUO and XIAOYAN ZHU
}

Department of Anesthesiology, Xiangya Hospital of Central South University, Changsha, Hunan 410008, P.R. China

Received December 4, 2017; Accepted March 30, 2018

DOI: $10.3892 /$ ijmm.2018.3613

\begin{abstract}
Treatment of neuropathic pain (NPP) continues to be a major challenge, and the underlying mechanisms remain to be elucidated. Previous studies have demonstrated that histone methylation is important in synaptic plasticity of the nervous system and may affect nuclear factor $-\kappa \mathrm{B}(\mathrm{NF}-\kappa \mathrm{B})$ signaling through epigenetic mechanisms. The present study aimed to investigate the role of Jumonji C domain 6 (JMJD6), a histone demethylase, in a chronic constriction injury (CCI) model of NPP. On the third day post-CCI surgery, a JMJD6 overexpressing lentiviral vector (LV-JMJD6) was intrathecally injected in the rats. Mechanical withdrawal threshold and thermal withdrawal latency were assessed prior surgery and on days 3, 7, 10 and 14 post-CCI. The results showed that intrathecal injection with the LV-JMJD6 attenuated CCI-induced pain facilitation. The expression of JMJD6 was lower following CCI surgery, and its expression was significantly increased following intrathecal injection with LV-JMJD6, compared with levels in normal saline (NS)- and negative control lentiviral vector (NC)-treated rats. The expression of spinal $\mathrm{NF}-\kappa \mathrm{B}$ phosphorylated (p-)p65 subunit and its downstream pain-associated effectors, including interleukin $1 \beta$ (IL-1 $\beta$ ), tumor necrosis factor- $\alpha$ (TNF- $\alpha)$ and vascular endothelial growth factor (VEGF), were increased following CCI surgery. Intrathecal injection with LV-JMJD6 suppressed activation of the p-p65 subunit in CCI rats. In addition, expression levels of its downstream effectors IL-1 $\beta$, TNF- $\alpha$ and VEGF were attenuated by intrathecal treatment with LV-JMJD6, compared with those in the NS- and NC-treated CCI rats. Furthermore,
\end{abstract}

Correspondence to: Dr Xiaoyan Zhu, Department of Anesthesiology, Xiangya Hospital of Central South University, 87 Xiangya Road, Changsha, Hunan 410008, P.R. China E-mail: zhuxiaoyan0402@gmail.com

Abbreviations: CCI, chronic constriction injury; GFP, green fluorescent protein; IL-1 $\beta$, interleukin-1 $\beta$; JMJD, Jumonji C domain; $\mathrm{NF}-\kappa \mathrm{B}$, nuclear factor- $\kappa \mathrm{B}$; NPP, neuropathic pain; TNF- $\alpha$, tumor necrosis factor- $\alpha$; VEGF, vascular endothelial growth factor

Key words: neuropathic pain, Jumonji C domain 6, nuclear factor-кB, tumor necrosis factor- $\alpha$, interleukin- $1 \beta$, vascular endothelial growth factor the JMJD6- and p65-immunoreactive cells overlapped in the spinal dorsal horn, however, co-immunoprecipitation showed that JMJD6 and the NF- $\mathrm{B}$ p65 subunit did not directly interact, indicating other functional connections may exist between these factors following CCI surgery. Collectively, these findings indicated an important mechanism underlying the pathogenesis of NPP. JMJD6 may exert its therapeutic

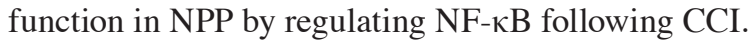

\section{Introduction}

The International Association for the Study of Pain defines neuropathic pain (NPP) as pain that is a direct outcome of trauma or diseases affecting the somatosensory nervous system (1). The clinical manifestations of NPP generally include spontaneous pain, hyperalgesia and paresthesia. NPP has become a public health problem, which affects a wide population globally (2). Extensive research has been performed to elucidate the neurobiological mechanisms of NPP, which is clinically managed by drugs, including opioids and calcium channel blockers. However, these therapies can be ineffective or can result in severe side effects $(3,4)$.

Evidence indicates that pain-related genes in sensory neurons exert a pivotal effect in the development and maintenance of NPP (5-7). Epigenetic mechanisms affect gene expression without changing the original DNA sequence. Studies have shown that epigenetic mechanisms are associated with synaptic plasticity, learning and memory (8). DNA methylation, histone modifications, including phosphorylation, acetylation, ubiquitination and methylation, and microRNA expression are involved in these epigenetic mechanisms. Histone modifications are closely linked with cell development, aging, and several other physiological and pathological processes. It has been shown that histone methylation affects synaptic plasticity within the nervous system, thus providing the basis for hyperalgesia (9).

Jumonji C domain (JMJD)-containing proteins form a large family of histone demethylases. The JMJD family member JMJD6 can catalyze the demethylation of arginine 2 on histone H3 and arginine 3 on histone H4 (10). Co-bound JMJD6 and bromodomain-containing protein 4 anti-pause enhancers may adjust the promoter proximal pause release of a series of transcription units through long-range interactions (11). In addition, JMJD6 can regulate estrogen receptor- $\alpha$ methylation, thereby regulating non-genomic estrogen signaling (12). As 
an epigenetic regulator, JMJD6 possesses a number of novel and unexpected nuclear functions, which remain to be fully elucidated (11).

Nuclear factor- $\kappa \mathrm{B}(\mathrm{NF}-\kappa \mathrm{B})$, a ubiquitous nuclear transcription factor, is reported to regulate numerous genes important for inflammation, central nervous system injury and NPP (13-15). Peripheral nerve injury can activate $N F-\kappa B$, thus upregulating the expression of proinflammatory cytokines, adhesion molecules and chemokines in the spinal cord, subsequently leading to NPP. Evidence indicates that arginine residues within $\mathrm{NF}-\kappa \mathrm{B}$ can be reversibly methylated by histone-modifying enzymes, including arginine methyl transferase and demethylase (16). Furthermore, several downstream genes can be activated by $\mathrm{NF}-\kappa \mathrm{B}$ methylation (16). It was found that the $\mathrm{p} 65$ subunit can be methylated at arginine 30 by protein arginine methyltransferase 5 in response to interleukin- $1 \beta$ (IL-1 $\beta$ ), which decreases the ability of NF- $\kappa \mathrm{B}$ to interact with the inhibited $\kappa \mathrm{B}$ elements and enhances gene expression (17). A number of studies have indicated that $\mathrm{NF}-\kappa \mathrm{B}$ can regulate the expression of tumor necrosis factor- $\alpha$ (TNF- $\alpha)$, IL- $1 \beta$ and vascular endothelial growth factor (VEGF); these mediators are also implicated in NPP $(18,19)$.

In the present study, it was hypothesized that JMJD6 may exert its epigenetic effects in NPP in chronic constriction injury (CCI) rats by regulating pain-associated mediators involved in the NF- $\kappa \mathrm{B}$ signaling pathway. The study evaluated the effects of the intrathecal administration of a JMJD6-overexpressing lentiviral vector (LV-JMJD6) on NPP through the mechanical withdrawal threshold (MWT), performed by stimulating the plantar surface of the left hind paw, and thermal withdrawal latency (TWL), performed by stimulating the same position. The effects on the expression of $\mathrm{NF}-\kappa \mathrm{B}$ and pain-associated factors, including TNF- $\alpha$, IL-1 $\beta$ and VEGF, were also examined in the CCI rats.

\section{Materials and methods}

Animals. Male Sprague-Dawley rats (220-250 g) were obtained from Central South University (Changsha, China) and housed in clean cages. The rats were allowed to adapt for 3 days and housed in controlled conditions $\left(22 \pm 0.5^{\circ} \mathrm{C}, 12: 12 \mathrm{~h}\right.$ dark/light cycle). All rats were provided with free access to food and water. All experiments were performed according to guidelines and protocols approved by the Animal Care Committee of Central South University, and were in accordance with the guidelines provided by the National Institute of Health. During surgical procedures, humane care was taken into consideration and animal suffering was minimized as much as possible.

CCI. The rats were anesthetized by inhalation of isoflurane (1-3\%) prior to the initiation of surgical procedures. The CCI model was implemented using the surgical procedures of Bennet and Xie (20). The left sciatic nerve was exposed using a blunt dissection technique. In the CCI group, four snug ligatures (4-0 chromic gut) were placed around the left sciatic nerve at an interval of $1.0 \mathrm{~mm}$ between ligatures under a microscope. Care was taken to tie the ligatures, so that the diameter of the sciatic nerve was gently constricted. The desired degree of constriction did not arrest the circulation thorough the superficial epineurial vasculature, and produced a small and brief twitch in the muscle surrounding the exposure. For the sham group, the sciatic nerve was exposed without ligation. To avoid variation, all the sham and nerve ligations were performed by the same individual, who was skilled in surgery. The incision was closed in layers. All rats were housed in cages with $3-5 \mathrm{~cm}$ of soft bedding following complete recovery from anesthesia.

Intrathecal catheter implantation. Under deep anesthesia, the rats were housed in a prone position. A longitudinal skin incision was made at the L4-5 intervertebral space in the midline of the back. Subsequently, the surrounding tissues were bluntly dissected and the intervertebral space was exposed. A lumbar intrathecal catheter (PE-10; Smiths Medical, Ashford, UK) was inserted into the subarachnoid space, as previously reported (21). Insertion of the catheter into the subarachnoid space was verified by the appearance of side tail swing or hind leg twitch. The success of catheter insertion was confirmed by definite outflow of cerebrospinal fluid.

Construction of lentiviral vectors. Based on sense and antisense sequences, a BLAST homology search was applied to guarantee that a single mRNA sequence of JMJD6 (NM_001012143) was targeted. To obtain the target gene fragment, polymerase chain reaction (PCR) primers were designed as follows: JMJD6 (1,253 bp), sense 5'-GAGGAT CCCCGGGTACCGGTCGCCACCATGAACCACAAGAGC AAGAAGC-3' and antisense 5'-TCCTTGTAGTCCATACCC CTGGAGGAACTGCGCTCTTTG-3'. PrimeSTAR HS DNA polymerase (cat. no. R010B, Takara Biotechnology Co., Ltd., Dalian, China) and primers (GeneRay Biotech Co., Ltd., Shanghai, China) were used for PCR amplification of JMJD6 cDNA. The PCR thermocycling conditions were as follows: Initial denaturation at $98^{\circ} \mathrm{C}$ for $5 \mathrm{~min}$; followed by 30 cycles at $98^{\circ} \mathrm{C}$ for $10 \mathrm{sec}, 55^{\circ} \mathrm{C}$ for $10 \mathrm{sec}, 72^{\circ} \mathrm{C}$ for $90 \mathrm{sec}, 72^{\circ} \mathrm{C}$ for 8 min and then maintained at $4^{\circ} \mathrm{C}$. The GV358, pHelper 1.0 and pHelper 2.0 vectors (Shanghai GeneChem Co., Ltd., Shanghai, China) were used as the lentivirus vectors. Plasmid DNA was extracted using the Plasmid DNA Extract kit (Qiagen, Inc., Valencia, CA, USA). Oligonucleotides were introduced into the plasmid GV358-JMJD6-green fluorescent protein (GFP), and the packaged plasmids were then cloned into the GV358-GFP lentiviral vector (Shanghai GeneChem Co., Ltd.). The recombinant vector and packaged plasmids were cotransduced into 293T cells (American Type Culture Collection, Manassas, VA, USA). The final titer of recombinant virus was $1 \times 10^{8} \mathrm{TU} / \mathrm{ml}$.

Treatments. The rats were randomly divided into four groups: Sham + normal saline (NS; $0.9 \%$ saline), CCI + NS, CCI + negative control lentiviral vector (NC) and CCI + LV-JMJD6. The treatment reagents $(20 \mu \mathrm{l})$ containing lentiviral titers of $1 \times 10^{8} \mathrm{TU} / \mathrm{ml}$ were administered via the lumbar intrathecal catheter on day 3 post-CCI. Following the administration of each reagent, $15 \mu \mathrm{l}$ of $0.9 \%$ saline was used to flush the catheter to avoid reagent residue. All experiments complied with the double-blind principle.

Assessment of pain behavior following CCI. The rats were inspected carefully for changes, including gait, posture of the affected hind paw, exercise ability and whether autophagy was present (20). Behavioral assessments were used to validate the 
success of CCI surgery. Only rats showing thermal hyperalgesia and mechanical allodynia were used in the following analysis. A 2390 Electronic von Frey anesthesiometer (IITC Life Science, Woodland Hills, CA, USA) was used to measure mechanical allodynia in all rats (22). Following placement of each rat into the plexiglas chamber, the MWT test was performed by stimulating the plantar surface of the left hind paw. Each Von Frey filament was held for $\sim 5 \mathrm{sec}$. A positive response was a rapid withdrawal of the hind paw during stimulation. When a positive response occurred, a filament with a lower force was used. If a negative response occurred, a filament with a higher force was used. A Hargreaves Tes7370 device (UgoBasile S.R.L, Comerio, Italy) was also applied to measure thermal hyperalgesia. The rats were placed on the surface of a glass plate, above which was a plexiglass chamber. Heat stimulation was positioned at the exposure site on the left hind paw. Each rat was assessed three times at intervals of 5 min. The MWT and TWL of each rat were assessed 1 day prior to CCI, and on days 3, 7, 10 and 14 following CCI surgery. To avoid variance, all behavioral assessments were performed by the same observer.

Total RNA isolation and reverse transcription-quantitative (RT-q)PCR analysis. On day 14 post-CCI, the rats were sacrificed and the lumbar spinal cord was removed. TRIzol reagent (Invitrogen; Thermo Fisher Scientific, Inc., Waltham, MA, USA) was used to extract total RNA. The $\mathrm{OD}_{260}$ of RNA was verified to calculate quantities of RNA using a spectrophotometer (Nanodrop 2000; Thermo Fisher Scientific, Inc.). Total RNA was reverse transcribed into cDNA using a reverse transcriptase kit (Takara Biotechnology Co., Ltd.) following the manufacturer's protocol and Oligo dT was used for the reverse transcription. The RT-qPCR conditions were as follows: $95^{\circ} \mathrm{C}$ for $5 \mathrm{~min}$, followed by 40 cycles of $94^{\circ} \mathrm{C}$ for $20 \mathrm{sec}, 56^{\circ} \mathrm{C}$ for $30 \mathrm{sec}$, and $72^{\circ} \mathrm{C}$ for $20 \mathrm{sec}$. All samples were run in duplicate. RT-qPCR was performed using a Viia ${ }^{\mathrm{TM}} 7$ Real-Time PCR System (Thermo Fisher Scientific, Inc.) and Quantifast SYBR Green PCR 2x Master Mix (Qiagen GmbH, Hilden, Germany). The following primers were used for amplification: IL-1 $\beta$, 5'-AGTCTGCACAGTTCCCCAAC-3' (forward) and 5'-TTA GGAAGACACGGGTTCCA-3' (reverse); $\beta$-actin, 5'-TCGTGC GTGACATTAAAGAG-3' (forward) and 5'-ATTGCCGAT AGTGATGACCT-3' (reverse); TNF- $\alpha, 5$ '-AGAGCCCCCAAT CTGTGTC-3' (forward) and 5'-TTCAGCGTCTCGTGTGTT TC-3' (reverse); VEGF, 5'-CACTGAGGAGTCCAACATCAC C-3' (forward) and 5'-CATCTCTCCTATGTGCTGGCCT-3' (reverse). $\beta$-actin was used as a control during PCR. SYBR Green assays were applied to measure mRNA expression, and relative expression levels of target mRNA were calculated using the $2^{-\triangle \Delta \mathrm{Cq}}$ method (23).

Western blot analysis. The L4-6 spinal cord segments were prepared for extraction of total protein. Tissues were homogenized in radioimmunoprecipitation assay lysis buffer (cat. no. P0013C; Beyotime Institute of Biotechnology, Haimen, China) containing $1 \mathrm{mM}$ phenylmethylsulfonyl fluoride (cat. no. ST506; Beyotime Institute of Biotechnology) and following centrifugation at $20,000 \times \mathrm{g}$ for $15 \mathrm{~min}$ at $4^{\circ} \mathrm{C}$, the supernatant was collected. The protein concentration was then determined using a BCA protein Assay Kit (Pierce; Thermo
Fisher Scientific, Inc.). A 10\% SDS-PAGE gel was used to separate the protein samples (50 $\mu \mathrm{g}$ of each sample), which were then transferred onto PVDF membranes. Subsequently, $5 \%$ non-fat dry milk blended with phosphate-buffered saline (PBS) and $0.05 \%$ Tween 20 was applied to block membranes for $1 \mathrm{~h}$, followed by incubation with mouse anti-JMJD6 (cat. no. SC-28348; 1:500; Santa Cruz Biotechnology Inc., Dallas, TX, USA), rabbit anti-NF-кB p-p65 (cat. no. ab86299; 1:2,000; Abcam, Cambridge, UK), rabbit anti-NF- $\kappa \mathrm{B}-\mathrm{p} 65$ (cat. no. 10745-1-AP; 1:1,500; ProteinTech Group, Inc., Chicago, IL, USA), rabbit anti-TNF- $\alpha$ (cat. no. AB1837P; 1:3,000; Merck Millipore, Darmstadt, Germany), rabbit anti-IL-1 $\beta$ (cat. no. AB1832P; 1:7,000; Merck Millipore), rabbit anti-VEGF (cat. no. 5365-100; 1:2,000, BioVision, Inc., Milpitas, CA, USA) or mouse anti- $\beta$-actin (cat. no. ab8226; 1:1,000; Abcam) primary antibodies at $4{ }^{\circ} \mathrm{C}$ overnight. The membranes were then incubated with goat anti-rabbit horseradish peroxidase (HRP)-IgG (cat. no. ab6721; 1:2,000; Abcam) or goat anti-mouse HRP-conjugated IgG (cat. no. ab205719; 1:2,000; Abcam) secondary antibodies for $1 \mathrm{~h}$ at room temperature. An ECL kit (Pierce; Thermo Fisher Scientific, Inc.) was used to visualize protein bands. Densitometric analysis was performed using Quantity One software version 4.1 (Bio-Rad Laboratories, Inc., Hercules, CA, USA). Protein levels were standardized to $\beta$-actin.

Double immunofluorescent labeling. Paraformaldehyde (4\%) was used to post-fix the spinal cord tissues of the CCI + LV-JMJD6 rats for $8 \mathrm{~h}$, following which the samples were embedded in paraffin and sections were cut at a thickness of $5 \mu \mathrm{m}$. To retrieve antigens and remove peroxidase activity, the deparaffinized sections were treated with $0.01 \mathrm{M}$ citrate buffer at $96^{\circ} \mathrm{C}$ for $1 \mathrm{~h}$, followed by $3 \% \mathrm{H}_{2} \mathrm{O}_{2}$ for $25 \mathrm{~min}$. The sections were then blocked with $10 \%$ donkey serum (cat. no. 017-000-121; Jackson ImmunoResearch Laboratories, Inc., West Grove, PA, USA) for $30 \mathrm{~min}$ and incubated with mouse monoclonal anti-JMJD6 antibody (1:50; Santa Cruz Biotechnology, Inc.) overnight at $4^{\circ} \mathrm{C}$, followed by incubation with red dihydroxyfluorene [Alexa Fluor 594-conjugated goat anti-mouse $\lg \mathrm{G}(\mathrm{H}+\mathrm{L})$; cat. no. SA00006-3; ProteinTech Group, Inc.] for $2 \mathrm{~h}$. The sections were then incubated with rabbit polyclonal anti-p65 antibody (1:100; ProteinTech Group, Inc.) overnight at $4^{\circ} \mathrm{C}$, and then with green dihydroxyfluorene [Alexa Fluor 488-conjugated Affinipure Goat anti-rabbit IgG (H+L); cat. no. SA00006-2; ProteinTech Group, Inc.] for $2 \mathrm{~h}$. Images of the double-labeled sections were captured with a Nikon microscope (Eclipse Ti-SR; Nikon, Tokyo, Japan).

Co-immunoprecipitation analysis. Spinal cord segments were prepared for the extraction of total protein. The samples were collected and solubilized in immunoprecipitation lysis buffer containing $25 \mathrm{mM}$ Tris, $150 \mathrm{mM} \mathrm{NaCl}, 1 \mathrm{mM}$ EDTA, $1 \%$ NP-40, $1 \%$ SDS, $0.5 \%$ Triton-X and 5\% glycerol (pH 7.4). The homogenates were incubated on ice for $15 \mathrm{~min}$ followed by centrifugation to remove insoluble material $(12,000 \mathrm{~g}$, $\left.4^{\circ} \mathrm{C}, 15 \mathrm{~min}\right)$. The lysates were incubated with a primary antibody $(2 \mu \mathrm{g} \mathrm{IgG})$, vs. normal IgG ( $2 \mu \mathrm{g}$, as a control) of the corresponding animal species overnight at $4^{\circ} \mathrm{C}$. Subsequently, $20 \mu \mathrm{l}$ of Protein A/G agarose beads (Santa Cruz Biotechnology, Inc.) was added to the antigen-antibody complex suspension 

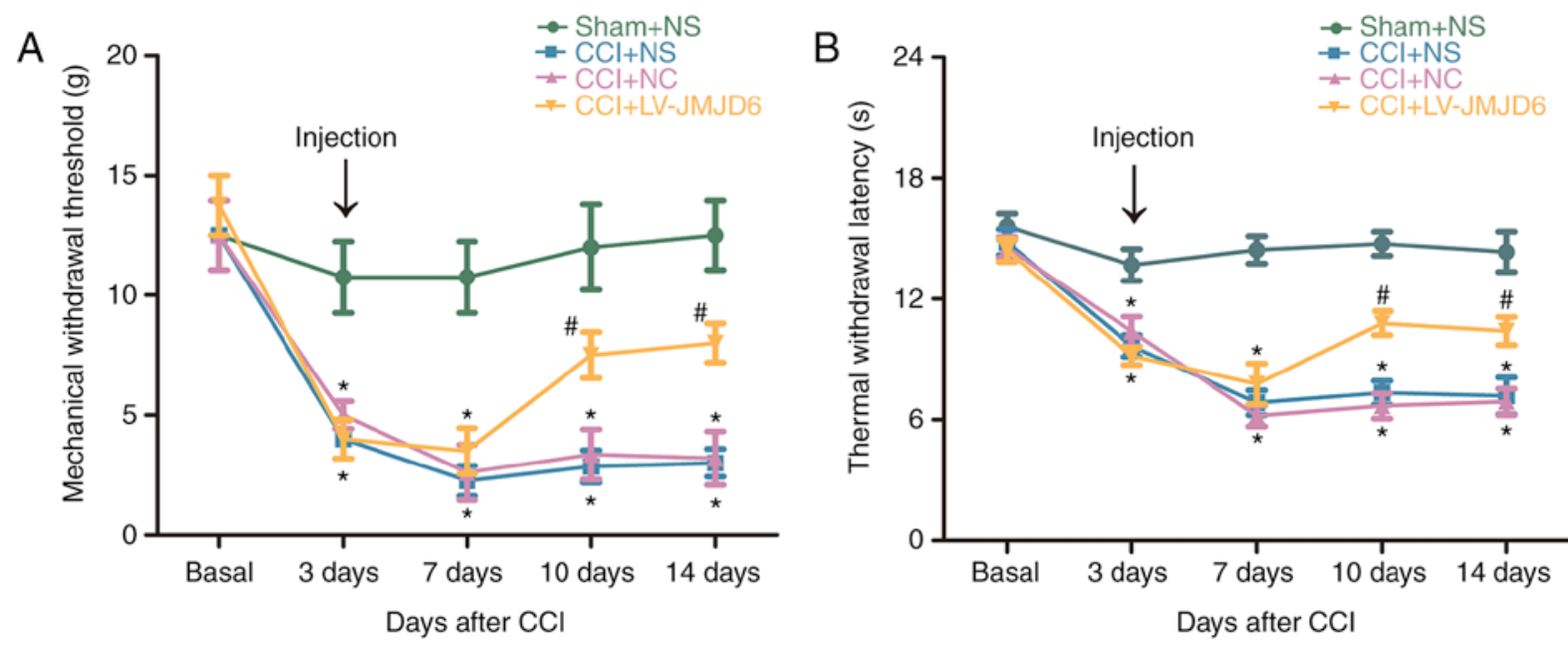

Figure 1. Effects of JMJD6 on behavioral changes in rats exposed to a CCI model of NPP. Animals were treated with a JMJD6-overexpressing lentiviral vector (LV-JMJD6) 3 days following induction of NPP via CCI. Control animals received sham surgery, NS or NC. (A) Changes in MWT (mean \pm standard error of the mean; $n=5$ ). (B) Changes in TWL (mean \pm standard error of the mean; $n=5$ ). Two-way analysis of variance was used as the statistical test. "P $<0.05$, vs. sham + NS-treated group; ${ }^{*} \mathrm{P}<0.05$, vs. CCI + NS-treated group and CCI + NC-treated group. CCI, chronic constriction injury; NPP, neuropathic pain; MWT, mechanical withdrawal threshold; TWL, thermal withdrawal latency; NS, normal saline; NC, negative control lentivirus.

and incubated for an additional $2 \mathrm{~h}$ at $4^{\circ} \mathrm{C}$ on a rocking platform. The immunocomplexes were then obtained and separated from other unbound proteins $\left(3,000 \mathrm{~g}, 4^{\circ} \mathrm{C}, 3 \mathrm{~min}\right)$, and washed three times with IP lysis buffer. $12 \%$ SDS-PAGE and PVDF membranes were used. The membranes were blocked with $5 \%$ non-fat dry milk in PBS with $0.05 \%$ Tween 20 for $1 \mathrm{~h}$. Primary antibody incubation with anti-JMJD6 (cat. no. SC-28348; 1:200; Santa Cruz Biotechnology) and anti-p65 (cat. no. 10745-1-AP; 1:500; ProteinTech Group, Inc.) was performed at room temperature on a shaker for $1 \mathrm{~h}$ and then at $4^{\circ} \mathrm{C}$ overnight, followed by incubation with horseradish peroxidase-conjugated goat anti-rabbit or goat anti-mouse secondary antibody (1:3,000; Santa Cruz Biotechnology, Inc.) for $1 \mathrm{~h}$ at room temperature. Protein brands were visualized by chemiluminescence using an ECL kit and quantified using Quantity One software.

Statistical analysis. Data are shown as the mean \pm standard error of the mean. For assessing mechanical and thermal hyperalgesia, two-way analysis of variance was used to compare the thresholds. For RT-qPCR and western blot analyses, one-way analysis of variance followed by the Student-Newman-Keuls post hoc test was applied using GraphPad Prism 5 (GraphPad Software, Inc., La Jolla, CA, USA). $\mathrm{P}<0.05$ was considered to indicate a statistically significant difference.

\section{Results}

JMJD6 alleviates CCI-induced behavioral changes. Following CCI surgery, the rats gradually showed typical signs of hyperalgesia and allodynia, including foot eversion, paw-licking and toe closing. The behaviors of the sham-operated rats were not altered markedly. CCI-induced mechanical allodynia and thermal hyperalgesia in the four groups are shown in Fig. 1A and B. For MWT and TWL prior to CCI, no significant difference was found between any of the groups
( $\mathrm{P}>0.05)$. Reductions in MWT and TWL in the CCI rats were detectable at $\sim 3$ days post-CCI $(\mathrm{P}<0.05)$, compared with the sham + NS-treated rats, indicating that the NPP model was successfully established. In the CCI + NS and CCI + NC rats, the MWT and TWL were reduced further on day 7 and continued to decrease until day 14 post-CCI, compared with those in the sham + NS rats $(\mathrm{P}<0.05)$. In the CCI + LV-JMJD6 group, MWT and TWL gradually recovered between day 10 and day 14, compared with that in the CCI + NS and CCI + NC groups $(\mathrm{P}<0.05)$. JMJD6 alleviated CCI-induced behavioral changes, suggesting a critical functional connection between JMJD6 and NPP (Fig. 1).

Effectiveness of lentiviral transfection in spinal dorsal horn cells. The effectiveness of lentiviral transfection on spinal cord tissue was detected on day 14 post-CCI. Lentiviral vectors, which carried a plasmid expressing GFP, were delivered into the subarachnoid space to transfect cells of the spinal cord (Fig. 2). GFP-positive cells were observed in the rats treated with LV-NC and LV-JMJD6, combined with the protein expression of JMJD6 (Fig. 3A and B), which indicated the success of lentiviral transfection.

Lentiviral vector-mediated JMJD6 increases expression of JMJD6 in CCI rats. The expression of JMJD6 was confirmed by western blot analysis (Fig. 3A and B). Compared with the sham + NS group, the expression of JMJD6 in CCI rats was significantly lower $(\mathrm{P}<0.05)$. Following $\mathrm{CCI}$, the rats were injected intrathecally with LV-JMJD6, the exogenous expression of JMJD6 caused by the overexpressing lentiviral vector elevated the level of JMJD6 and normalized the lower level of JMJD6 induced by CCI surgery. The expression of JMJD6 was upregulated in the CCI + LV-JMJD6 group, compared with that in its negative control group $(\mathrm{CCI}+\mathrm{NC}$ group; $\mathrm{P}<0.05)$ and solvent control group $(\mathrm{CCI}+\mathrm{NS}$ group; $\mathrm{P}<0.05)$. These results also indicated that the lentiviral vectors had transduced into the spinal cord successfully and the exogenous expression 

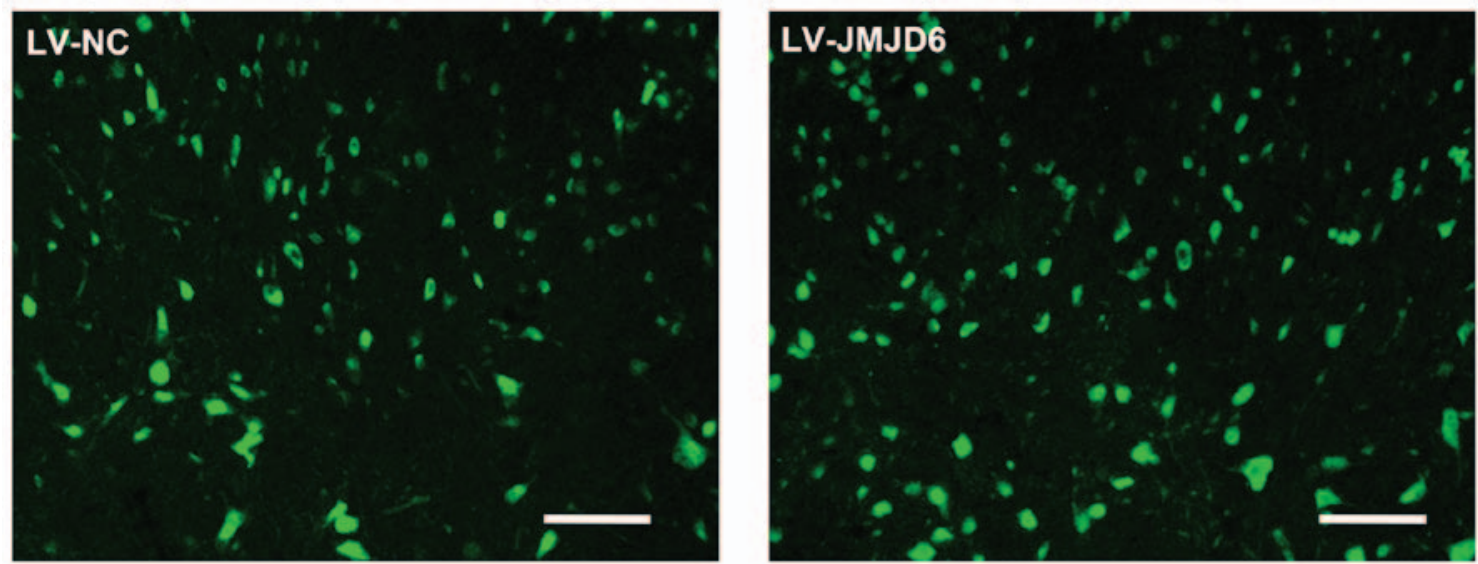

Figure 2. Effectiveness of lentiviral transfection. Fluorescence microscopy images of expression of GFP indicating the transfection of lentiviral vectors into cells of the spinal dorsal horn. GFP was detected in LV-NC-treated rats and LV-JMJD6-treated rats (scale bar=100 $\mu$ m). GFP, green fluorescent protein; $\mathrm{NC}$, negative control.
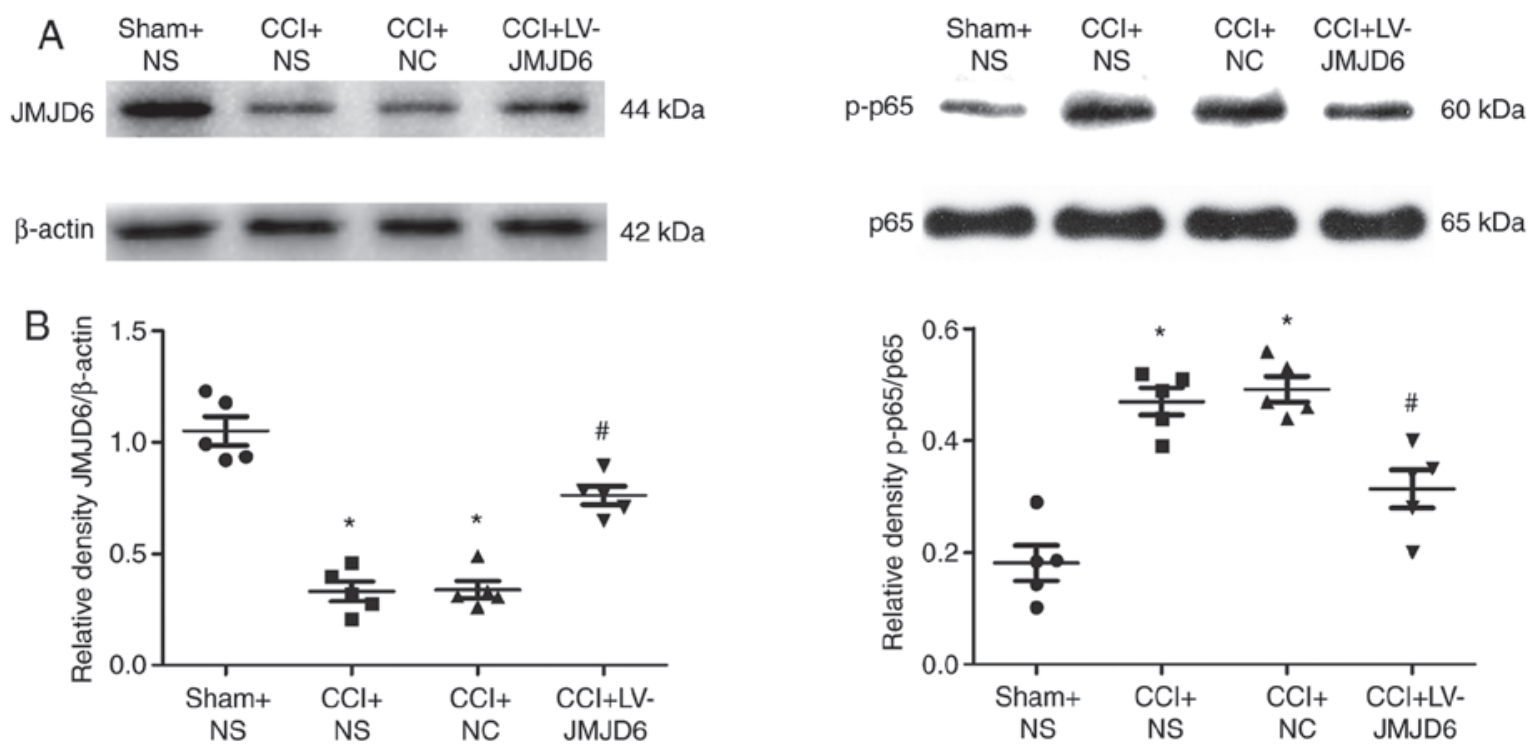

Figure 3. Changes in JMJD6 and nuclear factor- $\mathrm{BB}$ p-p65 subunit expression in rats exposed to a CCI model of NPP. Animals were treated with a JMJD6-overexpressing lentiviral vector (LV-JMJD6) 3 days following induction of NPP via CCI. Control animals received sham surgery, NS, or NC. Spinal cord L4-6 samples were prepared for western blot analysis 14 days following surgery. (A) Representative bands showing the protein expression of JMJD6 and p-p65. Expression of $\beta$-actin and p65 was used as a control. (B) Relative quantification of JMJD6/ $\beta$-actin and p-p65/p65 protein levels (mean \pm standard error of the mean; $\mathrm{n}=5$ ). One-way analysis of variance was used as the statistical tests. ${ }^{*} \mathrm{P}<0.05$, vs. sham $+\mathrm{NS}$ group; ${ }^{*} \mathrm{P}<0.05$, vs. CCI + NS and CCI + NC groups. CCI, chronic constriction injury; NPP, neuropathic pain; NS, normal saline; NC, negative control lentivirus; p-, phosphorylated.

of JMJD6 was attained. No difference in the expression level of JMJD6 was observed between the CCI + NS and CCI + NC groups $(\mathrm{P}>0.05)$.

JMJD6 attenuates CCI-induced expression of the $N F-\kappa B$ $p$-p65 subunit. The effects of JMJD6 on the expression of the NF-kB p-p65 subunit were verified by western blot analysis. Compared with the sham + NS group, the expression of the p-p65 subunit in the CCI rats was increased significantly $(\mathrm{P}<0.05$; Fig. $3 \mathrm{~A}$ and $\mathrm{B})$. However, intrathecal injection with LV-JMJD6 markedly attenuated the expression of the p-p65 subunit $(\mathrm{P}<0.05)$.

JMJD6 suppresses CCI-induced proinflammatory cytokine activation in spinal segments. RT-qPCR and western blot analyses were used to examine the mRNA and protein levels of proinflammatory cytokines, respectively. Compared with the sham + NS group, the results indicated that the expression levels of TNF- $\alpha$ and IL- $1 \beta$ were markedly increased in the $\mathrm{CCI}+\mathrm{NS}$ and $\mathrm{CCI}+\mathrm{NC}$ groups $(\mathrm{P}<0.05$; Fig. 4$)$. In the RT-qPCR (Fig. 4A) and western blot (Fig. 4B and C) analyses, JMJD6 attenuated the CCI-induced changes in proinflammatory cytokine expression, as demonstrated by reduced mRNA and protein levels of TNF- $\alpha$ and IL-1 $\beta(\mathrm{P}<0.05)$.

LV-JMJD6 injection decreases CCI-induced expression of $V E G F$. The mRNA and protein expression levels of VEGF were verified by RT-qPCR and western blot analyses, respectively (Fig. 5A-C). Compared with the sham + NS-treated group, the CCI rats exhibited significantly higher expression levels of 

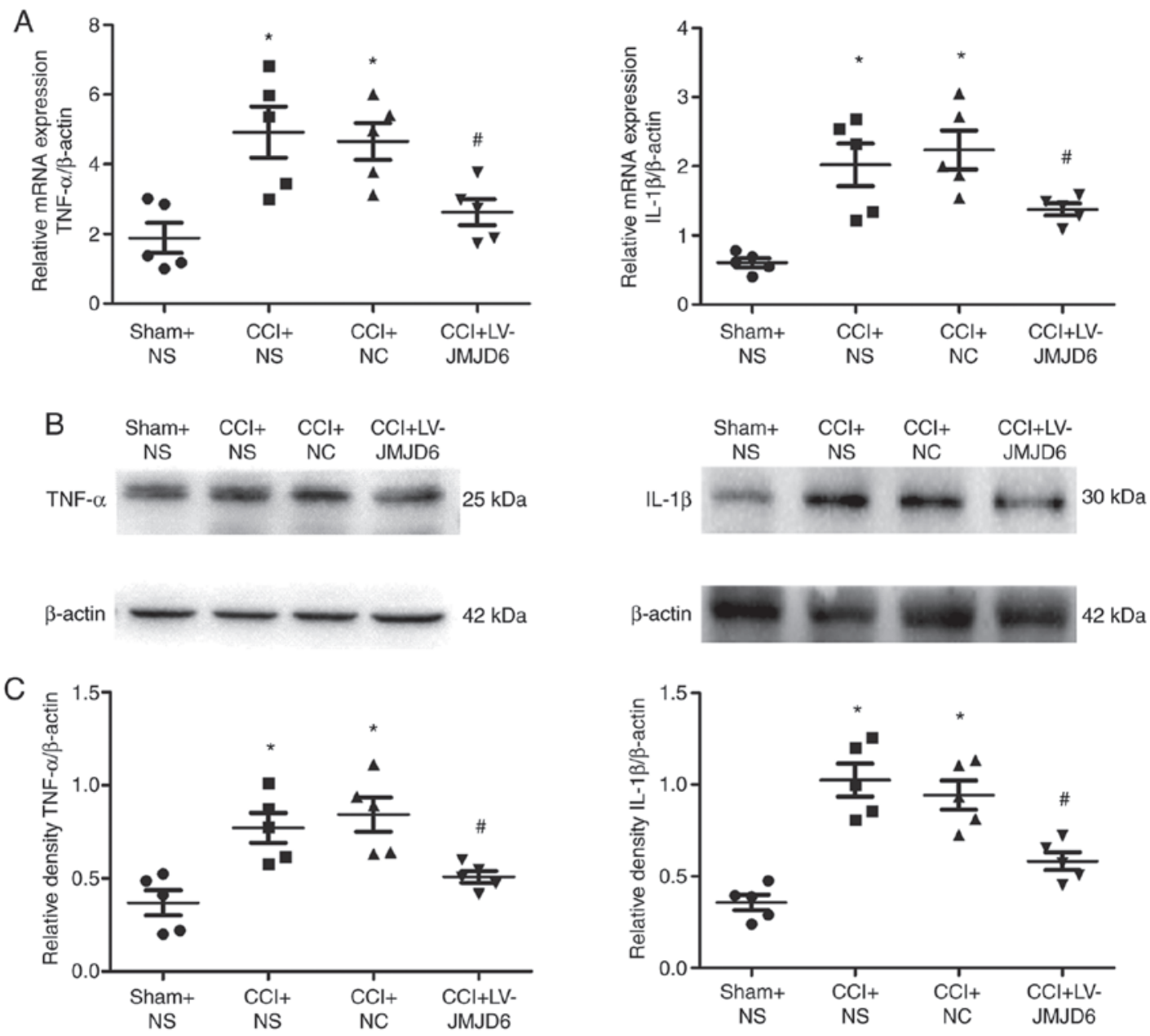

Figure 4. Effects of intrathecal injection with LV-JMJD6 on chronic constriction injury (CCI)-induced proinflammatory cytokine mRNA and protein expression. Animals were treated with a JMJD6-overexpressing lentiviral vector (LV-JMJD6) 3 days following induction of NPP via CCI. Control animals received sham surgery, NS, or NC. Spinal cord L4-6 samples were prepared for reverse transcription-quantitative polymerase chain reaction or western blot analyses 14 days following surgery. (A) Relative quantification of mRNA levels of TNF- $\alpha$ and IL-1 $\beta$ (mean \pm standard error of the mean; $n=5$ ). $\beta$-actin was used as a control. (B) Representative bands showing protein expression levels of TNF- $\alpha$ and IL-1 $\beta$. $\beta$-actin was used as a control. (C) Relative quantification of protein levels of TNF- $\alpha / \beta$-actin and IL-1 $\beta / \beta$-actin (mean \pm standard error of the mean; $n=5$ ). One-way analysis of variance was used as the statistical tests. ${ }^{*}<<0.05$, vs. sham + NS group; ${ }^{\prime} \mathrm{P}<0.05$, vs. CCI + NS and CCI + NC groups. CCI, chronic constriction injury; NPP, neuropathic pain; NS, normal saline; NC, negative control lentivirus; TNF- $\alpha$, tumor necrosis factor- $\alpha$; IL-1 $\beta$, interleukin-1 $\beta$.

VEGF $(\mathrm{P}<0.05)$. However, the expression of VEGF was lower following intrathecal injection of LV-JMJD6 $(\mathrm{P}<0.05)$.

Colocalization of JMJD6 and NF- $\kappa B$ p65 in the spinal cord. To assess the colocalization of JMJD6 and NF- $\mathrm{kB}$ p65 in the spinal cord of the CCI rats, double-labeling immunofluorescence was performed. Red fluorescence was used to label JMJD6-immunoreactive cells, green fluorescence was used to label p65-immunoreactive cells. JMJD6 and p65 double-positive cells (indicated in yellow in the merged image) in the spinal dorsal horn nuclei indicated that JMJD6 may exert its function together with p65 following CCI surgery (Fig. 6).

Absence of a direct interaction between JMJD6 and p65. The earlier experiments in the present study indicated the colocalization of JMJD6 and p65 in the spinal cord, however, whether JMJD6 and p65 interact directly remained to be elucidated. Therefore, co-immunoprecipitation analysis was used to determine their potential interactions. Specimens from two groups (CCI+LV-JMJD6, CCI+LV-NC) were detected. The results showed that 655 protein was not observed in co-immunoprecipitation with JMJD6, nor was JMJD6 protein observed in co-immunoprecipitation with p65 (Fig. 7), indicating that there may be no direct interactions between JMJD6 and p65.

\section{Discussion}

The CCI model, which is a classic model in NPP research, is achieved by placing loosely constrictive ligatures around the common sciatic nerve. The postoperative behaviors of CCI rats indicate that hyperalgesia, allodynia and, possibly, spontaneous pain were produced, which consistently represent signs in patients with NPP; CCI-induced hyperalgesia and allodynia normally present on day 3 post-surgery (24-26), which indicate a successfully established model. In the present study, treatments commenced on day 3 following CCI, at which point MWT and TWL were markedly decreased. In the LV-JMJD6-treated group, measurements in behavioral assessments, including mechanical allodynia and thermal hyperexcitability, recovered in a stepwise manner, compared 

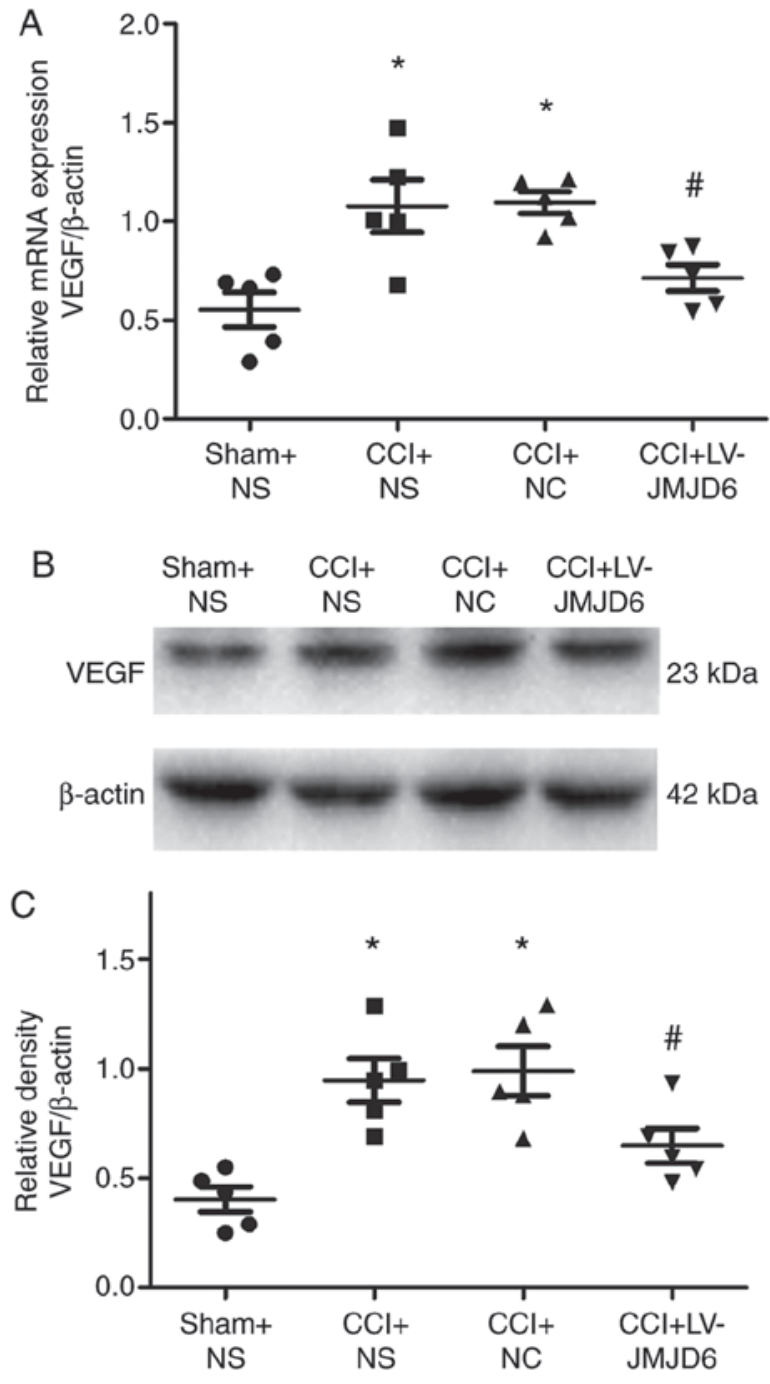

Figure 5. Effects of intrathecal injection with LV-JMJD6 on CCI-induced mRNA and protein expression of VEGF. Animals were treated with a JMJD6-overexpressing lentiviral vector (LV-JMJD6) 3 days following induction of NPP via CCI. Control animals received sham surgery, NS, or NC. Spinal cord L4-6 samples were prepared for reverse transcription-quantitative polymerase chain reaction or western blot analyses 14 days following surgery. (A) Relative quantification of mRNA levels of VEGF (mean \pm standard error of the mean; $n=5$ ). $\beta$-actin was used as a control. (B) Representative bands showing the protein expression level of VEGF. $\beta$-actin was used as a control. (C) Relative quantification of protein levels of VEGF/ $\beta$-actin 1 (mean \pm standard error of the mean; $n=5$ ). One-way analysis of variance was used as the statistical tests. ${ }^{*} \mathrm{P}<0.05$, vs. sham $+\mathrm{NS}$ group; ${ }^{\#} \mathrm{P}<0.05$, vs. CCI + $\mathrm{NS}$ and CCI + NC groups. CCI, chronic constriction injury; NPP, neuropathic pain; NS, normal saline; NC, negative control lentivirus; VEGF, vascular endothelial growth factor.

with those in the NC- and NS-treated CCI rats, and lasted until day 14, indicating that JMJD6 exerted an effect.

JMJD6 is known to be a demethylase, and has been implicated in an array of biological processes. Previous studies have found that JMJD6 and the serine/arginine-rich protein U2AF65 co-regulate a large number of alternative splicing events, which has important implications in development and disease processes (27). However, the function of JMJD6 in NPP remains to be elucidated. In the present study, the protein expression of JMJD6 in the spinal cord was reduced in the CCI rats. Intrathecal injection of the JMJD6-overexpessing lentiviral vector attenuated CCI-induced NPP and upregulated the protein expression of JMJD6, suggesting that JMJD6 may exert a function in NPP.

The NF- $\kappa \mathrm{B}$ pathway is pivotal in processes including cell regulation, differentiation and stress responses (28). The activation of $\mathrm{NF}-\kappa \mathrm{B}$ enhances gene expression by binding to target gene promoters (29). In several types of pain, $N F-\kappa B$ affects inflammatory responses and immunity by regulating genes encoding for chemokines, proinflammatory cytokines and adhesion molecules in the spinal cord (30). The inhibition of NF- $\kappa \mathrm{B}$ has been used to attenuate chronic pain states. Intrathecal injection of the $\mathrm{NF}-\kappa \mathrm{B}$ inhibitor pyrrolidine dithiocarbamate can alleviate CCI-induced allodynia (31). In the present study, expression of the NF- $\mathrm{B}$ p-p65 subunit in the spinal cord increased markedly following $\mathrm{CCI}$, consistent with previous findings that the activation of $\mathrm{NF}-\kappa \mathrm{B}$ is involved in or required for the induction of allodynia and hyperalgesia (29). In addition, expression of the NF- $\kappa \mathrm{B}$ p-p65 subunit decreased following intrathecal injection of LV-JMJD6, indicating that JMJD6 may suppress the activation of NF- $\kappa$ B.

Gene expression is epigenetically regulated by histone methyltransferases and demethylases via changing histone methylation states in several cellular processes, including differentiation and proliferation. The methylation states of numerous non-histone proteins can also be changed by modifiers, including effector proteins, which have key effects in cellular signaling networks (32). It has been noted that $\mathrm{NF}-\kappa \mathrm{B}$ signaling can be regulated by multiple post-translational modifications (33). NF- $\kappa \mathrm{B}$ can undergo arginine methylation, and R30 of p65 can be demethylated by PRMT5, resulting in the activation of NF- $\mathrm{B}$ signaling (17). As a demethylase, JMJD6 may regulate the effects of $\mathrm{NF}-\kappa \mathrm{B}$ in NPP. In the present study, the expression of NF- $\kappa \mathrm{B}$ p-p65 was examined following intrathecal injection of JMJD6. The results showed a marked increase in the expression of p-p65 following CCI; however, its activation was inhibited in the LV-JMJD6-treated CCI rats, indicating that JMJD6 may effectively inhibit the activity of $\mathrm{NF}-\kappa \mathrm{B}$ and alleviate NPP through epigenetic mechanisms.

Inflammatory cytokines are involved in the modulation of NPP. When tissues or nerves are damaged, proinflammatory cytokines (TNF- $\alpha$ and IL-1 $\beta$ ) are upregulated in the spinal cord (18). These cytokines are indicated to be downstream mediators of $\mathrm{NF}-\kappa \mathrm{B}$, and it has been reported that endoneural injection of an NF- $\mathrm{NB}$ inhibitor at the nerve lesion can attenuate hyperalgesia and downregulate cytokine mRNA levels at the site of injury (19). Therefore, in the present study, the expression levels of TNF- $\alpha$ and IL-1 $\beta$ were assessed to examine the effect of JMJD6 on NF- $\kappa \mathrm{B}$ downstream mediators. The majority of the findings suggested that the proinflammatory cytokines exerted their effects mainly in the early stages of pain. However, studies have also shown that central sensitization associated with NPP may result from nerve injury-induced cytokine release, suggesting long-term effects on nociceptive processing $(34,35)$. For example, following peripheral nerve injury, the expression of IL-1 $\beta$ continues to rise for at least 35 days (34). Furthermore, the neuronal release of cytokines can activate astrocytes, which can release a large number of cytokines and amplify pain signaling $(18,36)$. These findings are consistent with the results of the present study, which showed that the expression levels of TNF- $\alpha$ and IL-1 $\beta$ were significantly increased on day 14 post-CCI (Fig. 4). In 

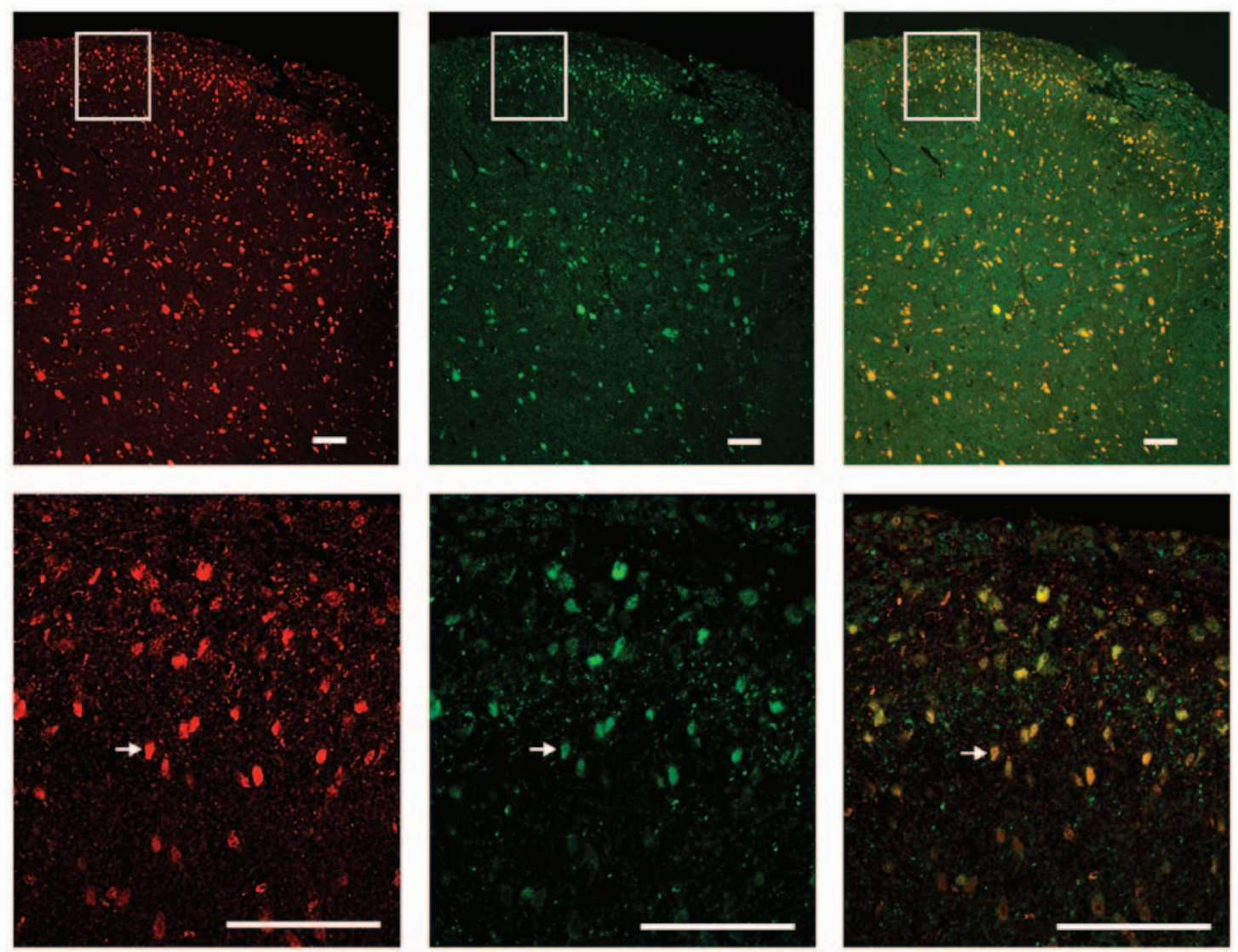

Figure 6. Colocalization of JMJD6 and NF-אB p65 in the spinal cord following CCI. Sections of L4-6 spinal cord at day 14 post-CCI were used in all experiments. Red fluorescence indicates JMJD6 immunoreactivity, green fluorescence indicates nuclear factor- $\mathrm{kB}$ p65 immunoreactivity. The images in lower panel are the enlarged images of the regions framed in the upper panel. Arrows indicate representative cells expressing both JMJD6 and p65 (scale bar $=75 \mu \mathrm{m})$. CCI, chronic constriction injury; JMJD6, Jumonji C domain 6.

Total protein IgG LV-JMJD6 LV-NC

$\operatorname{JMJD6}(44 \mathrm{kDa})$

Figure 7. Co-immunoprecipitation of JMJD6 and p65. IP of JMJD6 followed by western blot analysis for p65, and IP of p65 followed by western blot analysis for JMJD6. Antibodies applied in co-immunoprecipitation experiments were JMJD6 (1:200) and p65 (1:500). IP, immunoprecipitation; JMJD6, Jumonji C domain 6; NC, negative control.

the present study, the expression levels of TNF- $\alpha$ and IL-1 $\beta$ in the CCI rats were attenuated by intrathecal injection of LV-JMJD6, indicating that JMJD6 may affect the expression of downstream cytokines by regulating NF- $\mathrm{KB}$.

Changes in the expression of VEGF were also examined in the present study. VEGF can regulate vascular function through its effects on endothelial cells. Studies have shown that, in the dorsal root ganglia of CCI rats, VEGF immunoreactivity is enhanced (37). Treatment with an anti-VEGF antibody via intrathecal injection in rats attenuates reductions in MWT and TWL, which are induced by CCI surgery, and reduces the expression of VEGF receptors (37). Several studies

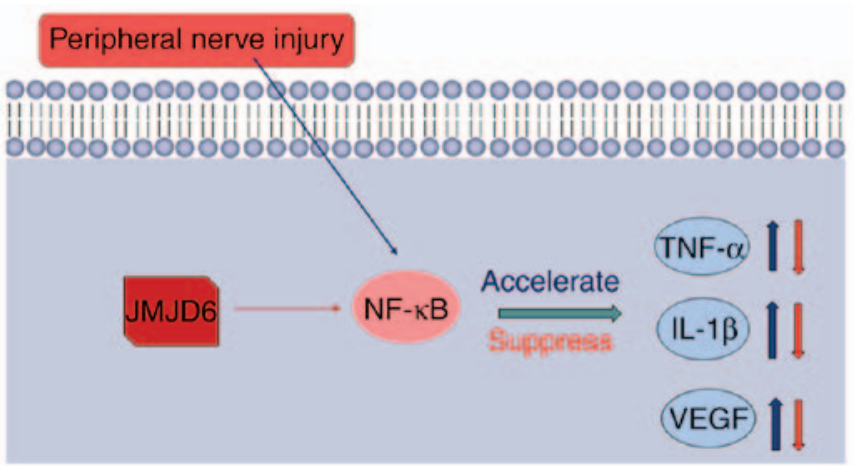

Figure 8. Model demonstrating the function of JMJD6 in regulating NF-kB during neuropathic pain. Peripheral nerve injury leads to the activation of NF- $\mathrm{KB}$ and enhancement of the transcription of NF- $\mathrm{KB}$ target genes TNF- $\alpha$, IL-1 $\beta$ and VEGF. JMJD6 in the nucleus may interact with NF- $\kappa B$ and, therefore, inhibit the transcriptional activation of NF- $\mathrm{kB}$. Blue arrows represent upregulation, whereas red arrows represent downregulation. JMJD6, Jumonji C domain 6; NF- $\kappa B$, nuclear factor-kB; TNF- $\alpha$, tumor necrosis factor- $\alpha$; IL-1 $\beta$, interleukin- $1 \beta$.

have shown that NF- $\kappa \mathrm{B}$ affects the expression of VEGF in various types of tumor. In addition, Gao et al showed that the NF- $\mathrm{KB}$ inhibitor, pyrrolidine dithiocarbamate hydrochloride, inhibited the expression of VEGF in tumor tissues (38). In the present study, the expression of VEGF was suppressed by JMJD6, which may be regulated by NF-кB. 
Based on the colocalization of JMJD6 and p65 observed in the present study, it was hypothesized that there may be a functional connection between them. JMJD6 and p65 colocalize within the nuclei in spinal dorsal horn, indicating that JMJD6 may regulate $\mathrm{NF}-\kappa \mathrm{B}$ within the nucleus and downregulate the transcription of mediators through a specific mechanism. However, the co-immunoprecipitation results in the present study indicated no direct interaction between JMJD6 and p65. According to the results, JMJD6 effectively relieved NPP of $\mathrm{CCI}$ rats, and downregulated the expression of $\mathrm{NF}-\kappa \mathrm{B}$ and its target genes, suggesting an intermediate biomolecule or signaling element may exist between JMJD6 and p65. It has been reported that TNF receptor-associated factor 6 can regulate NF- $\kappa \mathrm{B}$ and target genes through demethylation by JMJD6 in innate immune responses (39), however, this remains unclear in NPP. Therefore, future investigations are to focus on the epigenetic mechanism of JMJD6 at the cellular and animal levels, by assessing multiple time points in a long-term CCI model.

Based on the results of the present study, a novel model for the regulation by JMJD6 of NF- $\kappa \mathrm{B}$ in NPP was proposed (Fig. 8). Peripheral nerve injury leads to the activation of $N F-\kappa B$. NF- $\kappa B$ then binds to specific sites of target gene promoter regions to upregulate the expression of TNF- $\alpha$, IL-1 $\beta$ and VEGF, and other cytokines and adhesion molecules. However, JMJD6 in the nucleus may remove the methyl group from $N F-\kappa B$ and suppress the transcription of downstream mediators, thereby downregulating TNF- $\alpha$, IL- $1 \beta$ and VEGF, which may be the reason for the alleviation of NPP.

In conclusion, the results of the present study revealed that JMJD6 may exert key effects in NPP through regulating $\mathrm{NF}-\kappa \mathrm{B}$ following CCI. Following CCI, the overexpression of JMJD6 functionally reversed pain behavior, upregulated the spinal cord expression of JMJD6 and downregulated expression of the NF- $\kappa \mathrm{B}$ p-p65 subunit and its downstream mediators, including TNF- $\alpha$, IL-1 $\beta$ and VEGF. In addition, JMJD6 and p65 colocalized within the nucleus, although they did not interact directly, providing evidence for their potential functional contact. The results of the present study provide insights into the role of histone demethylation in NPP, which warrants further investigation. JMJD6 is expected to be a potential therapeutic target for NPP.

\section{Acknowledgements}

The authors would like to thank Dr Melony Black, Liwen Bianji and Edanz Group China (www.liwenbianji.cn/ac), for editing the English text in a manuscript draft.

\section{Funding}

This study was supported by grants from the National Natural Science Fund of China (grant nos. 81400916 and 81301043) and the Science and Technology Planning Project of Hunan Province, China (grant no. 2014SK3103).

\section{Availability of data and materials}

The datasets used and/or analyzed during the current study are available from the corresponding author on reasonable request.

\section{Authors' contributions}

QG and XZ designed and conceived the experiments; CW, MX and $\mathrm{CM}$ performed the experiments; $\mathrm{ZC}$ and $\mathrm{QG}$ analyzed the data; CW, MX and XZ contributed reagents, materials and analytical tools; CW and XZ wrote the manuscript.

\section{Ethics approval and consent to participate}

All experiments were performed according to guidelines and protocols approved by the Animal Care Committee of Central South University, and were in accordance with the guidelines provided by the National Institute of Health.

\section{Consent for publication}

Not applicable.

\section{Competing interests}

The authors declare that they have no competing interests.

\section{References}

1. Gilron I, Baron R and Jensen T: Neuropathic pain: Principles of diagnosis and treatment. Mayo Clin Proc 90: 532-545, 2015.

2. Jensen TS and Finnerup NB: Allodynia and hyperalgesia in neuropathic pain: Clinical manifestations and mechanisms. Lancet Neurol 13: 924-935, 2014.

3. Latremoliere A and Woolf CJ: Central sensitization: A generator of pain hypersensitivity by central neural plasticity. J Pain 10 : 895-926, 2009

4. Kerstman E, Ahn S, Battu S, Tariq S and Grabois M: Neuropathic pain. Handb Clin Neurol 110: 175-187, 2013.

5. Benarroch EE: Central neuron-glia interactions and neuropathic pain: Overview of recent concepts and clinical implications. Neurology 75: 273-278, 2010.

6. Zhu X, Li Q, Chang R, Yang D, Song Z, Guo Q and Huang C: Curcumin alleviates neuropathic pain by inhibiting p300/CBP histone acetyltransferase activity-regulated expression of BDNF and cox-2 in a rat model. PLoS One 9: e91303, 2014.

7. He Z, Guo Q, Xiao M, He C and Zou W: Intrathecal lentivirus-mediated transfer of interleukin-10 attenuates chronic constriction injury-induced neuropathic pain through modulation of spinal high-mobility group box 1 in rats. Pain Physician 16: E615-E625, 2013.

8. Descalzi G, Ikegami D, Ushijima T, Nestler EJ, Zachariou V and Narita M: Epigenetic mechanisms of chronic pain. Trends Neurosci 38: 237-246, 2015.

9. Kim SY, Levenson JM, Korsmeyer S, Sweatt JD and Schumacher A: Developmental regulation of Eed complex composition governs a switch in global histone modification in brain. J Biol Chem 282: 9962-9972, 2007.

10. Chang B, Chen Y, Zhao Y and Bruick RK: JMJD6 is a histone arginine demethylase. Science 318: 444-447, 2007.

11. Liu W, Ma Q, Wong K, Li W, Ohgi K, Zhang J, Aggarwal A and Rosenfeld MG: Brd4 and JMJD6-associated anti-pause enhancers in regulation of transcriptional pause release. Cell 155: 1581-1595, 2013.

12. Poulard C, Rambaud J, Hussein N, Corbo L and Le Romancer M: JMJD6 regulates ER $\alpha$ methylation on arginine. PLoS One 9: e87982, 2014.

13. Hoesel B and Schmid JA: The complexity of NF- $\mathrm{kB}$ signaling in inflammation and cancer. Mol Cancer 12: 86, 2013.

14. Gerondakis S, Fulford TS, Messina NL and Grumont RJ: NF-кB control of T cell development. Nat Immunol 15: 15-25, 2014.

15. Pan YD, Guo QL, Wang E, Ye Z, He ZH, Zou WY, Cheng ZG and Wang YJ: Intrathecal infusion of pyrrolidine dithiocarbamate for the prevention and reversal of neuropathic pain in rats using a sciatic chronic constriction injury model. Reg Anesth Pain Med 35: 231-237, 2010. 
16. Lu T and Stark GR: NF-кB: Regulation by methylation. Cancer Res 75: 3692-3695, 2015.

17. Wei H, Wang B, Miyagi M, She Y, Gopalan B, Huang DB, Ghosh G, Stark GR and Lu T: PRMT5 dimethylates R30 of the p65 subunit to activate NF- $\kappa$ B. Proc Natl Acad Sci USA 110: 13516-13521, 2013.

18. Ji RR, Berta T and Nedergaard M: Glia and pain: Is chronic pain a gliopathy? Pain 154 (Suppl 1): S10-S28, 2013.

19. Sakaue G, Shimaoka M, Fukuoka T, Hiroi T, Inoue T, Hashimoto N, Sakaguchi T, Sawa Y, Morishita R, Kiyono H, et al NF-kappaB decoy suppresses cytokine expression and thermal hyperalgesia in a rat neuropathic pain model. Neuroreport 12 : 2079-2084, 2001.

20. Bennett GJ and Xie YK: A peripheral mononeuropathy in rat that produces disorders of pain sensation like those seen in man. Pain 33: 87-107, 1988

21. Milligan ED, Hinde JL, Mehmert KK, Maier SF and Watkins LR: A method for increasing the viability of the external portion of lumbar catheters placed in the spinal subarachnoid space of rats. J Neurosci Methods 90: 81-86, 1999.

22. Cunha TM, Verri WA Jr, Valério DA, Guerrero AT, Nogueira LG, Vieira SM, Souza DG, Teixeira MM, Poole S, Ferreira SH and Cunha FQ: Role of cytokines in mediating mechanical hypernociception in a model of delayed-type hypersensitivity in mice. Eur J Pain 12: 1059-1068, 2008.

23. Livak KJ and Schmittgen TD: Analysis of relative gene expression data using real-time quantitative PCR and the 2(-Delta Delta C(T)) method. Methods 25: 402-408, 2001.

24. Zhu XY, Huang CS, Li Q, Guo QL, Wang Y, He X and Liao J: Temporal distribution of $\mathrm{p} 300 / \mathrm{CBP}$ immunoreactivity in the adult rat spinal dorsal horn following chronic constriction injury (CCI). Cell Mol Neurobiol 33: 197-204, 2013.

25. Zhang YQ, Guo N, Peng G, Wang X, Han M, Raincrow J, Chiu CH, Coolen LM, Wenthold RJ, Zhao ZQ, et al: Role of SIP30 in the development and maintenance of peripheral nerve injury-induced neuropathic pain. Pain 146: 130-140, 2009.

26. Zhu XY, Huang CS, Li Q, Chang RM, Song ZB, Zou WY and Guo Q: p300 exerts an epigenetic role in chronic neuropathic pain through its acetyltransferase activity in rats following chronic constriction injury (CCI). Mol Pain 8: 84, 2012.

27. Yi J, Shen HF, Qiu JS, Huang MF, Zhang WJ, Ding JC, Zhu XY, Zhou Y, Fu XD and Liu W: JMJD6 and U2AF65 co-regulate alternative splicing in both JMJD6 enzymatic activity dependent and independent manner. Nucleic Acids Res 45: 3503-3518, 2017.

28. Yao Z, Nie L, Zhao Y, Zhang Y, Liu Y, Li J and Cheng L: Salubrinal suppresses IL-17-induced upregulation of MMP-13 and extracellular matrix degradation through the NF- $\mathrm{BB}$ pathway in human nucleus pulposus cells. Inflammation 39: 1997-2007, 2016.
29. Luo JG, Zhao XL, Xu WC, Zhao XJ, Wang JN, Lin XW, Sun T and Fu ZJ: Activation of spinal NF-KB/p65 contributes to peripheral inflammation and hyperalgesia in rat adjuvant-induced arthritis. Arthritis Rheumatol 66: 896-906, 2014.

30. Lu H, Lei X and Zhang Q: Moderate activation of IKK2-NF-кB in unstressed adult mouse liver induces cytoprotective genes and lipogenesis without apparent signs of inflammation or fibrosis. BMC Gastroenterol 15: 94, 2015.

31. Yin Q, Fan Q, Zhao Y, Cheng MY, Liu H, Li J, Lu FF, Jia JT, Cheng W and Yan CD: Spinal NF- $\mathrm{B}$ and chemokine ligand 5 expression during spinal glial cell activation in a neuropathic pain model. PLoS One 10: e115120, 2015.

32. Alam H, Gu B and Lee MG: Histone methylation modifiers in cellular signaling pathways. Cell Mol Life Sci 72: 4577-4592, 2015.

33. Perkins ND: Post-translational modifications regulating the activity and function of the nuclear factor kappa B pathway. Oncogene 25: 6717-6730, 2006

34. Gustafson-Vickers SL, Lu VB, Lai AY, Todd KG, Ballanyi K and Smith PA: Long-term actions of interleukin-1beta on delay and tonic firing neurons in rat superficial dorsal horn and their relevance to central sensitization. Mol Pain 4: 63, 2008.

35. Echeverry S, Shi XQ and Zhang J: Characterization of cell proliferation in rat spinal cord following peripheral nerve injury and the relationship with neuropathic pain. Pain 135: 37-47, 2008.

36. Toyokawa G, Cho HS, Masuda K, Yamane Y, Yoshimatsu M, Hayami S, Takawa M, Iwai Y, Daigo Y, Tsuchiya E, et al: Histone lysine methyltransferase Wolf-Hirschhorn syndrome candidate 1 is involved in human carcinogenesis through regulation of the Wnt pathway. Neoplasia 13: 887-898, 2011.

37. Liu S, Xu C, Li G, Liu H, Xie J, Tu G, Peng H, Qiu S and Liang S: Vatalanib decrease the positive interaction of VEGF receptor-2 and $\mathrm{P} 2 \mathrm{X} 2 / 3$ receptor in chronic constriction injury rats. Neurochem Int 60: 565-572, 2012.

38. Gao P, Gao YJ and Liang HL: Effect of NF- $\kappa$ B inhibitor PDTC on VEGF and endostatin expression of mice with Lewis lung cancer. Asian Pac J Trop Med 8: 220-224, 2015.

39. Tikhanovich I, Kuravi S, Artigues A, Villar MT, Dorko K, Nawabi A, Roberts B and Weinman SA: dynamic arginine methylation of tumor necrosis factor (TNF) receptor-associated factor 6 regulates toll-like receptor signaling. J Biol Chem 290: 22236-22249, 2015. 